\title{
Human Umbilical Cord-Derived Mesenchymal Stem Cells Alleviate Acute Lung Injury Caused by Severe Burn via Secreting TSG-6 and Inhibiting Inflammatory Response
}

\author{
Xiaohong Hu $\mathbb{D},{ }^{1}$ Lingying Liu, ${ }^{2}$ Yu Wang, ${ }^{1}$ Yonghui Yu $\mathbb{D},{ }^{3}$ Zhongyuan Li, ${ }^{1}$ Yanan Liu, ${ }^{1}$ \\ and Jiake Chai $\mathbb{1}^{4}$ \\ ${ }^{1}$ Department of Pediatrics in the Fourth Medical Center, Chinese PLA General Hospital, Beijing 100048, China \\ ${ }^{2}$ Department of Nutrition in the Fourth Medical Center, Chinese PLA General Hospital, Beijing 100048, China \\ ${ }^{3}$ Beijing Engineering and Technology Research Center of Food Additives, Beijing Technology and Business University, \\ Beijing 100048, China \\ ${ }^{4}$ Department of Burn \& Plastic Surgery in the Fourth Medical Center, Chinese PLA General Hospital, Beijing 100048, China
}

Correspondence should be addressed to Xiaohong Hu; xhh304@163.com and Jiake Chai; cjk304@126.com

Received 15 December 2021; Revised 28 January 2022; Accepted 29 January 2022; Published 18 February 2022

Academic Editor: Stefania Cantore

Copyright (C) 2022 Xiaohong Hu et al. This is an open access article distributed under the Creative Commons Attribution License, which permits unrestricted use, distribution, and reproduction in any medium, provided the original work is properly cited.

Objectives. To investigate whether hUC-MSCs attenuated severe burn-induced ALI and the effects were based on TSG-6 secreted from hUC-MSCs. Method. A rat model was established and evaluated as follows: cytokine expression was measured by ELISA, and both inflammatory cell infiltration and lung injury were assessed by immunohistochemistry assay. Results. In vitro, TSG-6 levels in serum from the burn group were significantly increased compared with those from the sham group. In vivo, TSG-6 levels of lung tissues and serum in the burn+hUC-MSC group were significantly increased compared with those in the burn group. Both in lung tissues and in serum, increased levels of proinflammatory cytokines (TNF- $\alpha$, IL- $1 \beta$, and IL-6) were remarkably decreased, but the anti-inflammatory cytokine IL-10 increased after hUC-MSC administration $(p<0.05)$. These significant positive effects after hUC-MSC transplantation did not occur in the burn+siTSG-6 group. Conclusion. The intratracheal implantation of hUCMSCs has been an effective treatment for severe burn-induced ALI via promoting TSG-6 secretion and inhibiting inflammatory reaction in lung tissue.

\section{Introduction}

Acute lung injury (ALI) and its severe manifestation acute respiratory distress syndrome (ARDS) are common forms of hypoxic respiratory failure in patients with a mortality rate of $25-40 \%$ [1]. ALI/ARDS is also a major complication and a common cause of mortality in severely burned patients [2]. Growing evidence from experimental and clinical studies had shown that the systemic inflammatory response plays critical roles in the development and mortality of ALI/ARDS [3-5]. Several studies have shown that transplantation of MSCs can reduce the systemic inflammation, attenuate lung injury, and improve survival in models of ALI [6-8]. Unfortunately, there are no animal models of severe burn-induced ALI in these studies. The human umbilical cord MSCs (hUC-MSCs) emerged recently as an attractive source of MSCs for clinical application. The hUC-MSCs not only share the same features such as multilineage differentiation, paracrine functions, and immunemodulatory properties of all MSCs but also have some unique advantages, such as not requiring for bone marrow matching, low immunogenicity, higher self-renewal capacities, and accelerating injury tissue repair processes $[9,10]$. Our previous experiments had demonstrated that severe burns could bring a systemic inflammatory reaction. Meanwhile, hUC-MSCs could alleviate the systemic inflammatory reaction of severe burn through secreting a variety of bioactive factors [11]. However, little is known about hUC-MSCs in the prevention of severe burn-induced ALI. The multipotential anti-inflammatory protein tumor necrosis factor- 
(TNF- $\alpha$-) stimulated gene/protein 6 (TSG-6) has been found to be efficacious in a wide range of disease models, including respiratory disease models. Our previous study further testified the potential role and mechanism of hUC-MSCs secreting TSG-6 that attenuated severe burn-induced excessive inflammation via inhibiting activation of P38 and JNK signaling ways $[11,12]$. Above discoveries prompted us to further investigate whether hUC-MSCs attenuate severe burninduced ALI via paracrine secreting TSG-6, which inhibits the inflammatory reaction in lung tissue. Within this study, we investigated the therapeutic potential and mechanism of hUC-MSCs on severe burn-induced ALI in a rat model.

\section{Materials and Methods}

2.1. hUC-MSC Preparation. All experimental procedures were in accordance with the international guidelines for biomedical research in experimental animals issued by the Committee of International Organizations of Medical scientific (CIOMS). We approved the certification of the institutional animal care and use committee of the Fourth Medical Center of PLA General Hospital and obtained the informed consent of all subjects. The primary human umbilical cord mesenchymal stem cells (hUC-MSCs) were purchased from ScienCell Research Laboratories (ScienCell, San Diego, CA). The hUC-MSCs were examined using flow cytometry and are positive for CD105, CD90, CD44, CD29, and HLA-I and negative for CD34, CD45, HLA-DR, CD31, and $\mathrm{vWF}$ as described in our previous publication [12]. They have the potential to develop into mature cells that produce fat, cartilage, bone, tendons, and muscle. These properties, in combination with their developmental plasticity, have generated tremendous interest because of the potential use of MSC in regenerative medicine to replace damaged tissues.

2.2. Ex Vivo Evaluation of TSG-6 Released from hUC-MSCs in Serum of Burn Animals. The hUC-MSCs were plated into 6 -well plates with a cell density of 5000 cells $/ \mathrm{cm}^{2}$ and incubated with $3 \mathrm{ml}$ mesenchymal stem cell medium serum free (MSCM-sf) (ScienCell Research Laboratories, San Diego, CA) for 1 day, which were subsequently cultured continuously with $3 \mathrm{ml}$ of MSCM-sf containing $10 \%$ sham serum or burn serum at 24 hours postsevere burn. Currently, the levels of inflammatory mediators, oxidative stress factors, and other factors were very high. TSG-6 secretion from hUC-MSCs after $10 \%$ burn serum continuous culture and the supernatant of the cells were assayed by using the ELISA kit.

2.3. hUC-MSCs Were Transfected with siRNA for TSG-6. We selected the lentiviral expression vector containing the TSG6 siRNA sequence (GeneChem, Shanghai, China) for specific targeting TSG-6, including Lenti-TSG-6-siRNA, and its negative control vector, Lenti-GFP-siRNA. TSG-6 siRNA lentivirus vectors were generated by ligating the vector PGCLV. TSG-6 siRNA oligonucleotide sequences are forward $5^{\prime}$ CCGGTTCTCCGAACGTGTCACGTTTCAAGAGAACGT GACACGTTCGGAGAATTTTTG-3' and reverse $5^{\prime}$-AATT
CAAAAATTCTCCGAACGTGTCACGTTCTCTTGAAA CGTGACACGTTCGGAGAA-3'.

The sequences of control siRNA are forward $5^{\prime}$-CCGG GCAAGCTGACCCTGAAGTTCATCTCGAGATGAACT TCAGGGTCACGTTGCTTTTTG- $3^{\prime}$ and reverse $5^{\prime}$-AATT CAAAAAGCAAGCTGACCCTGAAGTTCATCTCGAGA TGAACTTCAGGGTCACGTTGC-3'.

The recombinant lentivirus was produced by cotransfecting HEK293T cells with pGC-LV-GFP-siRNA or pGCLV-TSG-6-siRNA, pHelper 1.0, and pHelper 2.0 plasmids using Lipofectamine 2000 (GeneChem, Shanghai, China). The hUC-MSCs were transfected with the prepared lentivirus (Lenti-TSG-6-siRNA or Lenti-GFP-siRNA). The hUCMSCs were plated into either 6-well plates or T-75 culture bottles with a cell density of 5000 cells $/ \mathrm{cm}^{2}$ and incubated with $3 \mathrm{ml}$ or $10 \mathrm{ml} \mathrm{MSCM}$-sf without antibiotics for 1 day. And then, these cells were transfected with $1 \mathrm{ml}$ medium of Lenti-TSG-6-siRNA or Lenti-GFP-siRNA according to the protocol provided by the manufacturer. After 12 hours, the medium was changed, and these cells were incubated with MSCM-sf without antibiotics for 3-4 days sequentially. The efficiency of TSG-6 gene knockdown in hUC-MSCs was evaluated using GFP-fluorescence observation and Western blot. Then, hUC-MSCs in T-75 culture bottles were harvested with $0.25 \%$ trypsin and resuspended at $5 \times 10^{6}$ cells in $1 \mathrm{ml}$ sterile PBS for subsequent implantation.

2.4. Animals. All studies adhered to procedures consistent with the International Guiding Principles for Biomedical Research Involving Animals issued by the Council for the International Organizations of Medical Sciences (CIOMS), and all experimental protocols were approved by the Institutional Animal Care and Use Committee at the Fourth Medical Center of PLA General Hospital and were performed in accordance with the AVMA Guidelines for the Euthanasia of Animals: 2013 Edition. Six-week-old male Wistar rats (180$220 \mathrm{~g}$ ) were obtained from the local animal facility and housed at the Institute of Animal Experiments of the Fourth Medical Center of PLA General Hospital in stables with a temperature of $22^{\circ} \mathrm{C}$, a relative humidity of $55 \%$, and a day/night cycle of 12/12 hours, with food and water ad libitum throughout the experiment.

2.5. Experimental Groups. Wistar rats were randomly divided into 5 groups: (1) sham, (2) burn, (3) burn+hUCMSCs (burn and implantation with hUC-MSCs), (4) burn+vehicle (burn and implantation with hUC-MSCs transfected with Lenti-GFP-siRNA), and (5) burn+siTSG-6 (burn and implantation with hUC-MSCs transfected with Lenti-TSG6-siRNA). Rats in each group were divided equally into three subgroups of 6 rats according to the indicated time points of $\mathrm{d} 1, \mathrm{~d} 3$, and $\mathrm{d} 7$ for the samples collected.

2.6. Animal Treatment. The rats were anesthetized with Avertin $(20 \mathrm{mg} / \mathrm{ml}$ and $300 \mathrm{mg} / \mathrm{kg}$ ) (2,2,2-tribromoethanol, Sigma, USA) via intraperitoneal injection. The dorsal and abdominal hair was completely shaved. Rat models with $50 \%$ TBSA full-thickness burn injury were established via placing the backside and abdomen into hot water $\left(94^{\circ} \mathrm{C}\right)$ 
for $12 \mathrm{~s}$ and $6 \mathrm{~s}$, respectively $[11,13,14]$. Intraperitoneal injections of balanced salt solution $(40 \mathrm{ml} / \mathrm{kg})$ were immediately administered to prevent shock in all groups. We adopted a 20-gauge central catheter as a tracheal catheter, while rats were restrained on the $45^{\circ}$ inclined operating board with their head facing the operator. The upper incisors of the rat were fixed with a cotton thread to the nails at one end of the operating board, so the head of the rat was raised, and we inserted the laryngoscope into its mouth. The glottis of the rat can be seen in the light of the laryngoscope. The tracheal catheter was quickly inserted into the airway when the glottis was opened $[15,16]$. The rats in the burn+hUC-MSC group immediately received intratracheal implantation of $5 \times 10^{6}(1 \mathrm{ml})$ hUC-MSCs after burn. The rats in the burn+vehicle and burn+siTSG- 6 groups received intratracheal implantation of $5 \times 10^{6}(1 \mathrm{ml})$ hUCMSCs transfected with Lenti-GFP siRNA or Lenti-TSG-6siRNA at the same time. The rats in the sham and burn groups received intratracheal implantation of $1 \mathrm{ml}$ PBS. All rats in each group were clinically evaluated. The burn wound and rats in the sham group were treated as previously described [11]. The blood and lung tissue samples were collected at each indicated time point. After anesthesia with the above anesthetics, the rats' blood was extracted from the inferior vena cava. And then, rats were euthanized with an overdose of pentobarbital sodium intravenously.

At each indicated time point, blood samples from the inferior vena cava were extracted, and lung tissue samples were collected. And then, rats were euthanized with an overdose of pentobarbital sodium intravenously.

2.7. Specimen Collection and Detection. Both the lung tissue and blood samples in all groups were taken from the aorta ventralis at $d 1, d 3$, and $d 7$ after injury. Subsequently, one piece of lung specimen was stored in liquid nitrogen for TSG-6 detection using the ELISA kit; also, another piece was fixed using $4 \%$ paraformaldehyde for immunohistochemical analysis and $\mathrm{H} \& \mathrm{E}$ staining. The detection range of the kit is from $0.312 \mathrm{ng} / \mathrm{ml}$ to $20 \mathrm{ng} / \mathrm{ml} .100 \mathrm{mg}$ lung tissue was rinsed with PBS, homogenized in $1 \mathrm{ml}$ of PBS, and stored overnight at $-20^{\circ} \mathrm{C}$. After two freeze-thaw cycles were performed to break the cell membranes, the homogenates were centrifuged for 5 minutes at $5000 \mathrm{~g}$ at $2-8^{\circ} \mathrm{C}$. The supernatant was removed and assayed immediately. The standard was diluted following the manufacturer's instruction. Use the stock solution to produce a 2 -fold dilution series. Mix each tube thoroughly before the next transfer. The undiluted standard serves as the high standard ( $20 \mathrm{ng} /$ $\mathrm{ml})$. The sample diluent serves as the standard zero.

2.8. Histological Analyses. After fixation with $4 \%$ paraformaldehyde for 1 week at room temperature, the specimens were embedded in paraffin and sectioned into five-micrometerthick sections. Then, the slices were deparaffinized with dimethyl benzene, rehydrated, and then stained with $\mathrm{H} \& \mathrm{E}$ in accordance with standard procedures. Infiltrations of neutrophils and macrophages were detected via incubating with specific antibodies (MPO, CD68; from R\&D Systems, Minneapolis, MN), the corresponding secondary antibody, and the PAP (peroxidase-antiperoxidase) complex in turn and finally stained with DAB (3,3'-diaminobenzidine). Myeloperoxidase (MPO) exists in the cytoplasm of neutrophils and can be used as an indicator of neutrophils in lung tissue. The positive reaction of neutrophils is brown-yellow colorization. CD68 is a macrophage-specific antigen which is direct evidence for the identification of macrophages. The positive reaction of macrophages is brown colorization. Randomly, five different visual fields under a light microscope (400x) of each respective section were chosen, and CD86and MPO-positive cells were counted by an experienced scientist in a blinded manner.

2.9. Western Blotting. Protein from hUC-MSCs was isolated using RIPA buffer (Sigma Life Science), containing PMFS (Sigma Life Science) and complete Mini Protease inhibitor cocktail (Roche). Cells were centrifuged at $3000 \mathrm{~g}$ for $10 \mathrm{~min}$ to collect the nuclei, and then, the supernatants were further centrifuged at $17,000 \mathrm{~g}$ for $20 \mathrm{~min}$ to separate the mitochondrion. The cytoplasm was diluted in Laemmli buffer and denaturized at $95^{\circ} \mathrm{C}$ for $10 \mathrm{~min}$. Protein was quantified using a commercial bicinchoninic acid (BCA) kit (BCA Protein Assay Kit; Pierce Biotechnology Inc., Rockford, IL, USA). Equal amounts of protein were subjected to SDS-PAGE gel as previously described [17], and the antiTSG-6 antibody $(1: 1,000)$ and anti- $\beta$-actin antibody $(1: 20,000)$ (R\&D Systems, Minneapolis, MN) were used for the protein expression assay.

2.10. Enzyme-Linked Immunosorbent Assay (ELISA). $100 \mathrm{mg}$ lung tissue was rinsed and homogenized and then stored overnight at $-20^{\circ} \mathrm{C}$. The samples were performed to break the cell membranes; the homogenates were centrifuged for 5 minutes at $5000 \mu \mathrm{g}$ at $2-8^{\circ} \mathrm{C}$. The supernatant was removed. The double-antibody sandwich ELISA kits (eBioscience, USA) were used to detect the levels of tumor necrosis factor alpha (TNF- $\alpha$ ), interleukin- $1 \beta$ (IL- $1 \beta$ ), IL-6, IL-10, MPO, and TSG-6 in lung tissues and serum according to the manufacturer's protocols.

2.11. Statistical Analysis. All data were expressed as the mean \pm SD and analyzed using SPSS 18.0 (SPSS Inc., Chicago, IL, USA). ANOVA followed by the Tukey Cicchetti test for multiple comparisons or nonparametric KruskalWallis test as appropriate was used for statistical analysis. Statistical significance level was set at $p<0.05$.

\section{Results}

3.1. TSG-6 Levels in Supernatant/Lung Tissues/Serum and TSG-6 Knockdown Efficiency. To investigate whether TSG6 secreted from hUC-MSCs influenced their therapeutic effect or not, we firstly examined the levels of TSG-6 secretion from hUC-MSCs after treatment with serum of sham or burn. Compared with the sham group, TSG-6 levels in the supernatant of burn serum were significantly increased in vitro at 24 hours postburn (Figure 1(a)). The hUCMSCs cultured in vitro were transfected with Lenti-GFP siRNA or Lenti-TSG-6-siRNA. As shown in Figure 1(b), representative photographs demonstrated successful 


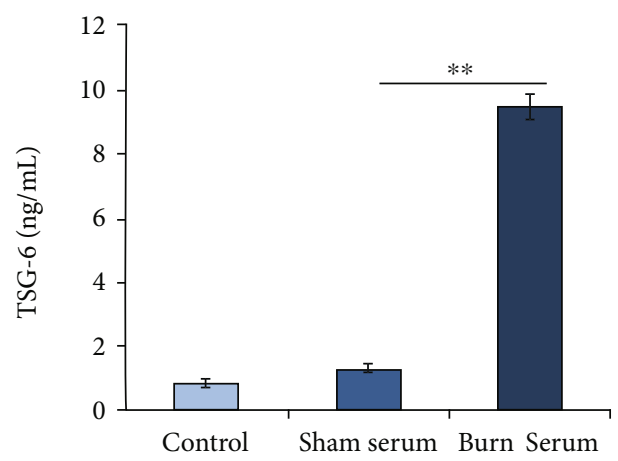

(a)

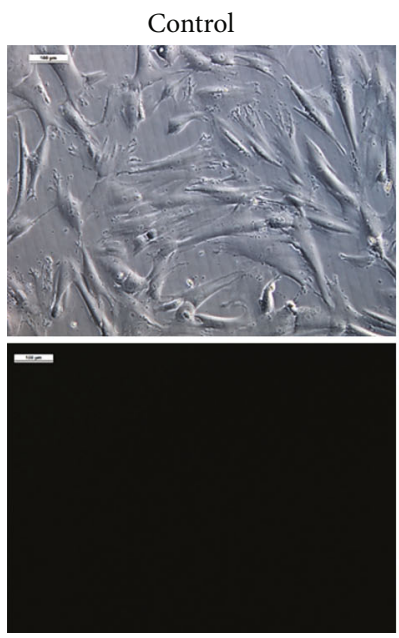

Vehicle

siTSG-6
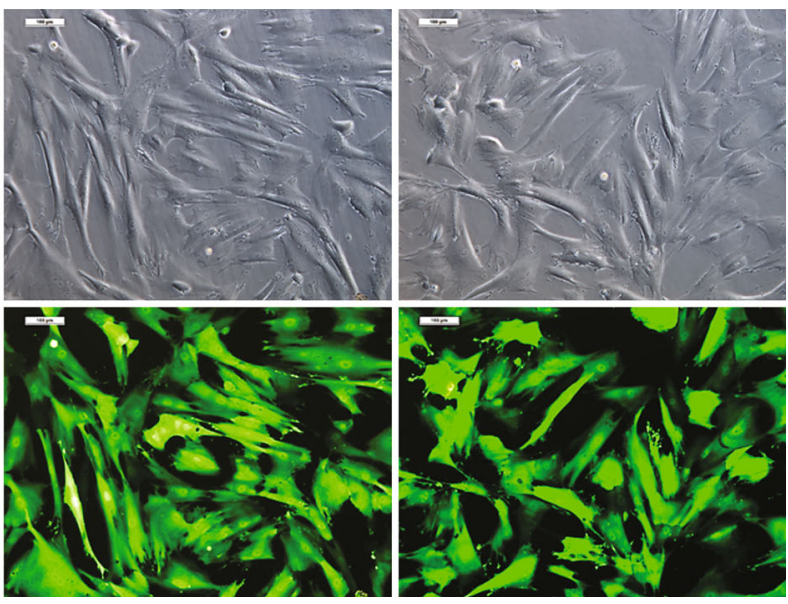

(b)

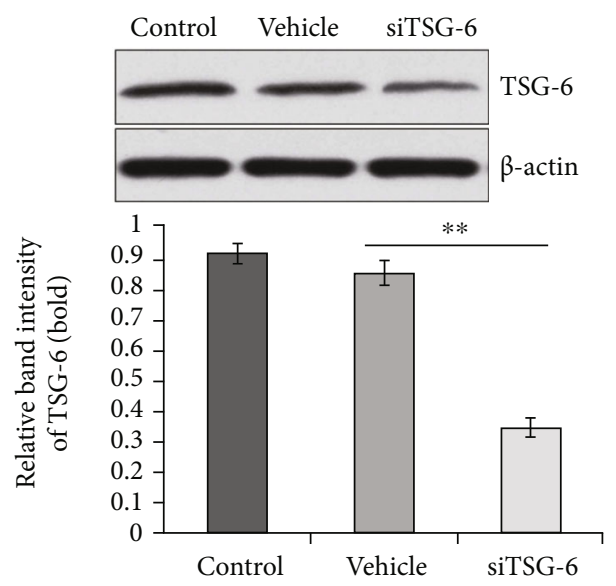

(c)

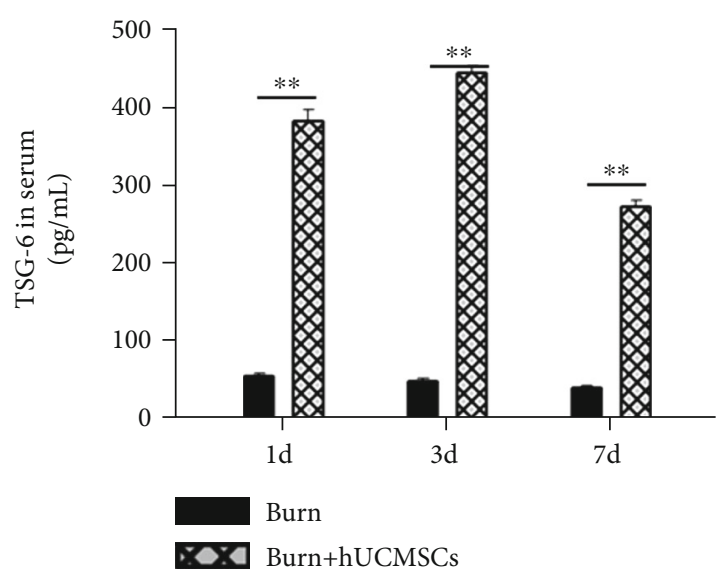

(d)

Figure 1: Continued. 


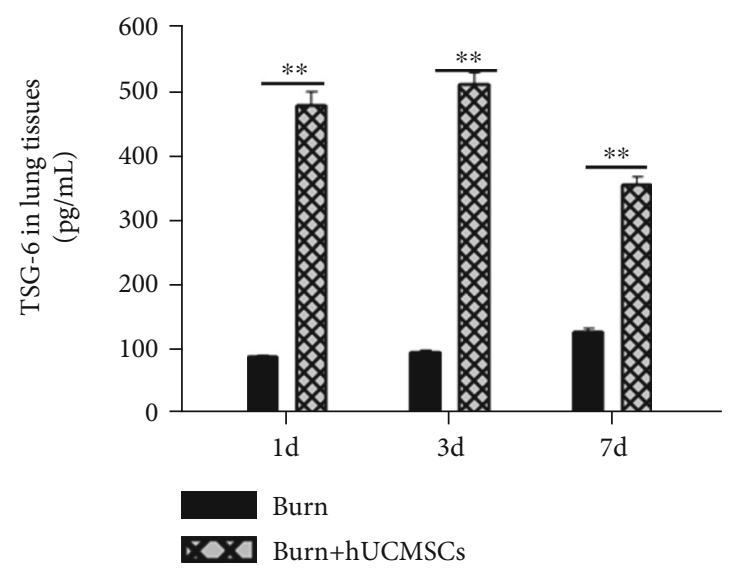

(e)

FIGURE 1: Secretion and knockdown efficiency of the TSG-6. (a) TSG-6 levels in supernatants of control, sham serum, and burn serum groups were evaluated by ELISA. Compared with control and sham groups, the TSG-6 levels in the burn serum group were significantly increased. (b) Representative photographs demonstrated successful transfection with Lenti-GFP-siRNA (vehicle) and Lenti-TSG-6-siRNA (siTSG-6) in hUC-MSCs (the inverted fluorescence microscope, 100x). (c) The levels of TSG-6 protein expression in hUC-MSCs after transfection with Lenti-GFP-siRNA and Lenti-TSG-6-siRNA were detected by Western blotting, and quantitative analysis is shown in the corresponding histogram. (d, e) TSG-6 levels in lung tissue and serum of burn and burn+hUC-MSC groups were also evaluated using ELISA. TSG-6 level of the burn+hUC-MSC group was significantly higher than that of the burn group. Values are represented as mean $\pm \mathrm{SD}(n=6)$. Asterisk $(*)$ and double asterisk $(* *)$ stand for $p<0.05$ and $p<0.01$, respectively.

transfection with Lenti-GFP siRNA or Lenti-TSG-6-siRNA. The data from Western blotting showed that TSG-6 expression was significantly decreased after Lenti-TSG-6-siRNA transfection compared with that of the vehicle group (Figure 1(c)). In vivo, TSG-6 levels of the burn+hUC-MSC group in lung tissues were significantly increased compared with those of the burn group at $\mathrm{d} 1, \mathrm{~d} 3$, and $\mathrm{d} 7$ postsevere burn (Figure 1(d)). Meanwhile, TSG-6 levels of the burn+hUC-MSC group in serum were significantly increased compared with those in the burn group in vivo (Figure 1(e)). TSG-6 levels of lung tissues and serum reached the peak at d3 in the burn+hUC-MSC group.

3.2. The Effect of hUC-MSCs and TSG-6 Knockdown on Structural Damage and Total Inflammatory Cell Infiltration in Lung Tissues after Severe Burn. We evaluated the therapeutic influence of hUC-MSCs and TSG-6 knockdown on the structural changes and infiltrated degree of total inflammatory cells in the lungs at $\mathrm{d} 1, \mathrm{~d} 3$, and $\mathrm{d} 7$ postsevere burn using $H \& E$ staining. As shown in Figure 2, compared with the sham group, the structural damage, including alveoli fusion, interstitial hemorrhage, alveoli exudation, and infiltrated degree of total inflammatory cells in the lung tissues of the burn group, was remarkably increased at d1. Meanwhile, these changes were less severe in the burn+hUCMSC group at d1. Destroyed structure and inflammatory cell infiltration after burn, such as neutrophil infiltration, interstitial edema, and pulmonary hemorrhage, were all improved gradually at $\mathrm{d} 3$ and $\mathrm{d} 7$. However, we noted that burn+hUC-MSCs recovered faster than other groups. Moreover, we found that the therapeutic effect of hUC-MSCs on the structural damage and total inflammation was sharply decreased after TSG-6 knockdown (Figure 2). Respectively, we calculated the neutrophil and macrophage infiltration of lung tissues induced by severe burns to evaluate the therapeutic influence of hUC-MSCs and TSG-6 knockdown on lung tissues at $\mathrm{d} 1, \mathrm{~d} 3$, and $\mathrm{d} 7$ after severe burns using immunohistochemical staining. As shown in Figure 3, compared with the sham group, the neutrophil infiltration degrees of lung tissues in the burn group were remarkably increased, and they were markedly decreased by hUC-MSC administration $(p<0.05)$. Furthermore, the data showed that the therapeutic effect of hUC-MSCs on neutrophil infiltration in the lung was sharply decreased after TSG-6 knockdown (Figure 3(a)). The quantitative analysis was reported in the corresponding histogram $(p<0.05)$ (Figure $3(\mathrm{~b}))$. As shown in Figure 4, in comparison with those in the sham group, macrophage infiltration degrees of lung tissues in the burn group were significantly increased, which were dramatically decreased after hUC-MSC implantation $(p<0.05)$. Furthermore, the data showed that the therapeutic effect of hUCMSCs on macrophage infiltration in the lung was also sharply decreased after TSG-6 knockdown (Figure 4(a)). The quantitative analysis was reported in the corresponding histogram $(p<0.05)$ (Figure 4(b)). It suggested that the therapeutic effect of hUC-MSCs on attenuating burn-induced neutrophil and macrophage infiltration was dependent on the level of TSG-6 expression. Downregulation of TSG-6 expression in hUC-MSCs significantly reduced their therapeutic function on improving structural damages and total inflammatory cell infiltrations of the lung tissues (Figures 2-4).

3.3. The Effect of hUC-MSCs and TSG-6 Knockdown on Inflammatory Cytokines in Lung Tissues after Severe Burn. We also tested the levels of the proinflammatory cytokines TNF- $\alpha$, IL- $1 \beta$, IL- 6 , and MPO and the anti-inflammatory cytokine IL-10 in lung tissues at $\mathrm{d} 1, \mathrm{~d} 3$, and $\mathrm{d} 7$ after severe 


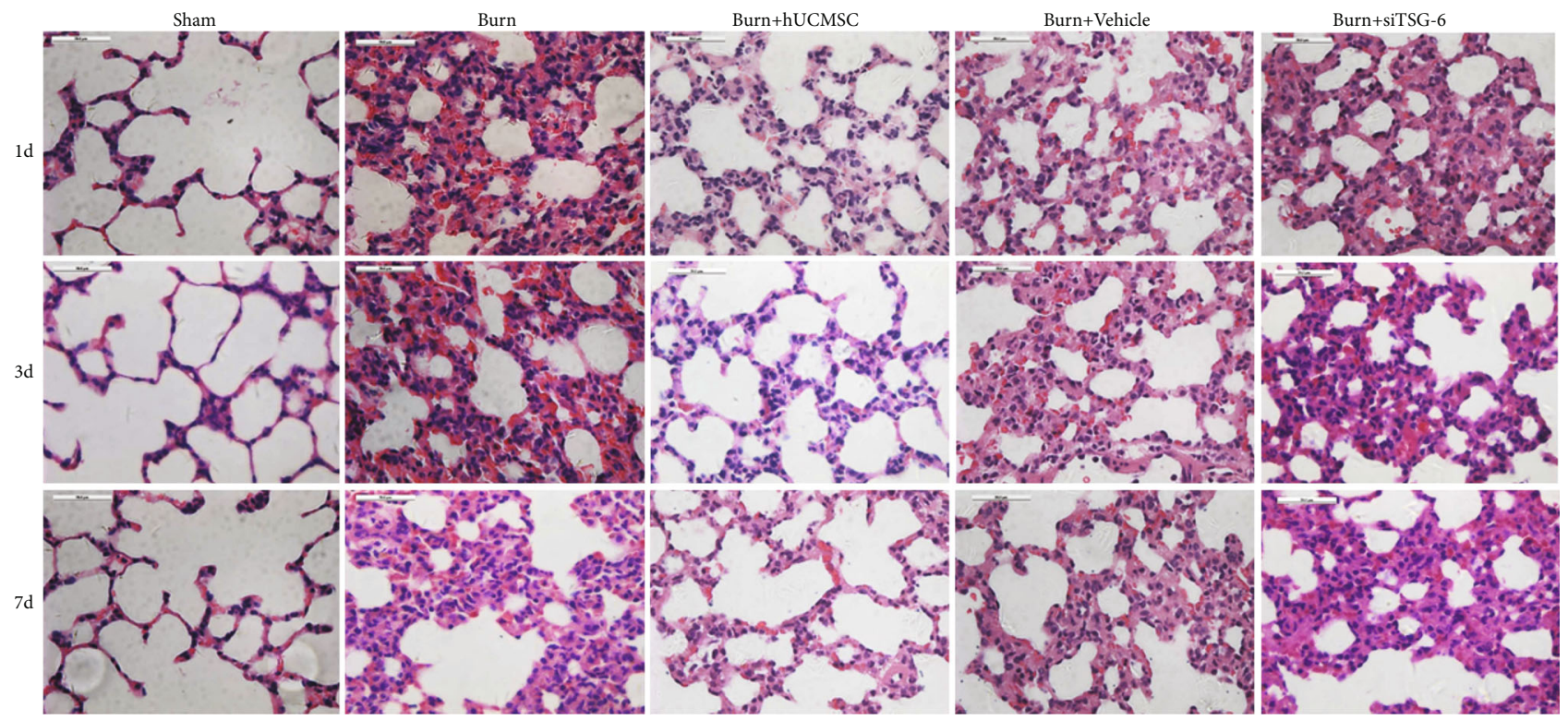

Figure 2: Effect of TSG-6 on the structural damage and total inflammatory cell infiltrations of lung tissues induced by severe burn. Representative photographs of lung tissues in all groups using H\&E staining are shown (the light microscope, 400x).

burn using ELISA. As shown in Figure 5, compared with the sham group, the levels of proinflammatory cytokines, such as TNF- $\alpha$ (Figure 5(a)), IL-1 $\beta$ (Figure 5(b)), IL-6 (Figure 5(c)), and MPO (Figure 5(d)), in the lung tissues of the burn group were remarkably increased, and they were all markedly decreased after hUC-MSC administration $(p<0.05)$. It is worth noting that the level of the antiinflammatory cytokine IL-10 (Figure 5(e)) in the burn+hUC-MSC group was significantly higher than that in the burn group and sham group, and that in the burn group was also higher than that in the sham group $(p<0.05)$.

While the data also indicated that the therapeutic effect of hUC-MSC knockdown TSG-6 surely increased the levels of proinflammatory cytokines, such as TNF- $\alpha$, IL- $1 \beta$, IL- 6 , and MPO, and decreased the anti-inflammatory cytokine IL-10 in lung tissues $(p<0.05)$ (Figure 5).

\section{Discussion}

During the last few years, human MSCs as a cellular therapy for the ALI/ARDS have ignited much interest of researchers, and the therapeutic effects have been demonstrated in different animal models [18-20]. Studies have shown that MSCs have therapeutic effects in various preclinical models of lung diseases via direct differentiation and paracrine action [21-23]. Indeed, there were a few of MSCs that survive for more than one week after systemic administration, suggesting that the main effects of MSCs are probably mediated by paracrine mechanisms [24].

Patients with ALI caused only by severe burns or scald were not uncommon in the clinic, especially in children. In the present study, we established a severe burn-induced ALI in a rat model to investigate the therapeutic effects of hUC-MSCs and their possible mechanism. We found that intratracheal implantation of hUC-MSCs increased the sur- vival rates and significantly attenuated severe burn-induced ALI via inhibiting pulmonary inflammation, and the therapeutic effects of hUC-MSCs were strongly reduced when the expression of TSG- 6 was inhibited via RNA interference. It indicated that the therapeutic effects of hUC-MSCs in severe burn-induced ALI rats were associated with the soluble factor TSG-6 secreted by hUC-MSCs itself.

We created a rat model with a classical pulmonary inflammatory state via severe burn $[13,14,25]$. The severity of the lung injury was characterized by several pathological parameters, just as described in the diagram (Figures 2-4). All these pathological changes are closely tied to the human ALI, which indicates that our model successfully duplicated human ALI. These parameters were all improved after intratracheal implantation of hUC-MSCs, which indicated the therapeutic benefits of hUC-MSC implantation on severe burn-induced ALI. These findings proved that the intratracheal injection of hUC-MSCs attenuated lung injury in severe burn-induced ALI rats. Our results were consistent with some other previous studies, in which the administration of hUC-MSCs or BM-MSCs reduced systemic inflammation and attenuated LPS-induced ALI in rats [26].

ALI is an uncontrollable pulmonary inflammatory disease characterized by the release of cytokines and neutrophil accumulation. Neutrophils are the primary cells being convened to the site of inflammation and releasing inflammatory cytokines. Macrophages are another predominant cells existing in either a proinflammatory (M1) or antiinflammatory (M2) state, acting as a trigger of the inflammatory response, and contributing to both the initiation and resolution of inflammation. MSC treatment could regulate macrophages with the anti-inflammatory phenotype and simultaneously enhance the phagocytic activity of macrophages [27]. In our study, the infiltration of neutrophils and macrophages in lung tissue in the burn group was 


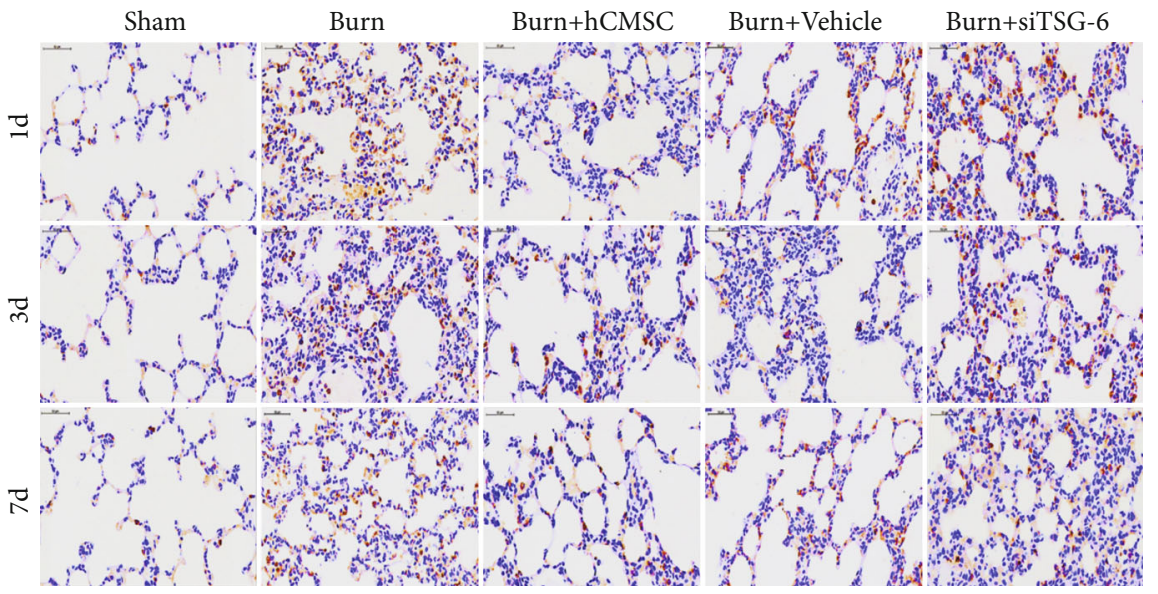

(a)

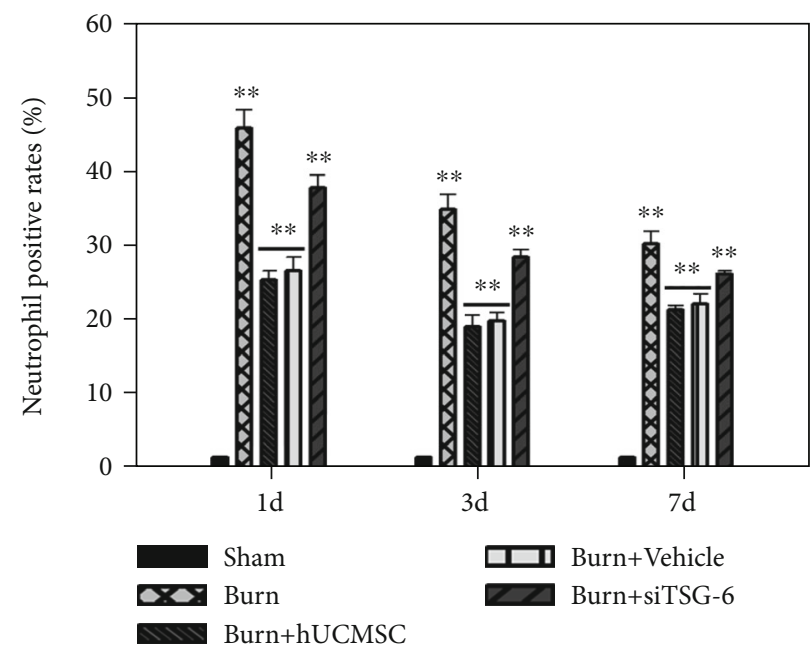

(b)

Figure 3: Effect of TSG-6 secretion and knockdown on neutrophil infiltrations of lung tissues induced by severe burn. (a) Representative photographs of $\mathrm{MPO}^{+}$in lung tissues using immumohistochemical staining are shown (the light microscope, 400x). (b) The quantitative analysis is shown in the corresponding histogram. Values are represented as mean $\pm \mathrm{SD}(n=6)$. Asterisk $(*)$ and double asterisk $(* *)$ stand for $p<0.05$ and $p<0.01$, respectively.

remarkably increased and surely reduced after being treated with hUC-MSCs. This alleviative lung injury of hUC-MSCs was once confirmed in canine radiation-induced ALI model via reducing oxidative stress, inflammatory reactions, and TGF- $\beta$-Smad $2 / 3$ pathway activation [28].

MPO is an enzyme stored in neutrophils and macrophages and released into the extracellular fluid in the setting of the inflammatory process. Since MPO is an important enzyme in the inflammatory process, there is an ongoing interest in the use of MPO as a biomarker for assessing the extent of inflammatory response in our present study. After a severe burn, a remarkable increase in MPO in lung tissues was detected in the burn group, and the peak level was shown at d1. As we expected, treatment with hUC-MSCs clearly reduced the MPO activity at $\mathrm{d} 1, \mathrm{~d} 3$, and $\mathrm{d} 7$. Previously, Li et al. found that intravenous infusion with hUCMSCs significantly inhibited LPS-stimulated MPO activity in rat lung tissues [26]. Reduced MPO activity indicates an improvement of lung injury and confirmed the therapeutic effects of hUC-MSCs on burn-induced lung injury through promoting anti-inflammatory homeostasis.

The proinflammatory cytokines TNF- $\alpha$, IL- $1 \beta$, and IL- 6 are the primary mediators of the acute phase response. Previous studies showed that the decreased neutrophil recruitment into the lung tissue and suppressed expression of TNF- $\alpha$, IL- $1 \beta$, and IL- 6 can improve the outcomes of ALI [29]. Several other studies have also shown that MSCs could decrease the plasma level of TNF- $\alpha$ and IL- $1 \beta$ through paracrine secretion [30]. Consistent with previous studies, increased levels of these inflammatory cytokines were found in our model of severe burn-induced ALI. After severe burns, the concentration of TNF- $\alpha$, IL- $1 \beta$, and IL- 6 significantly increased. The difference is simply that the peak level occurs at different times. For TNF- $\alpha$ and IL- $1 \beta$, the peak level appeared on d1, while the peak level of IL-6 appeared on $\mathrm{d} 3$. When hUC-MSCs were intratracheally implanted after burn, the concentration of proinflammatory cytokines in lung tissues decreased significantly, which was mostly in 


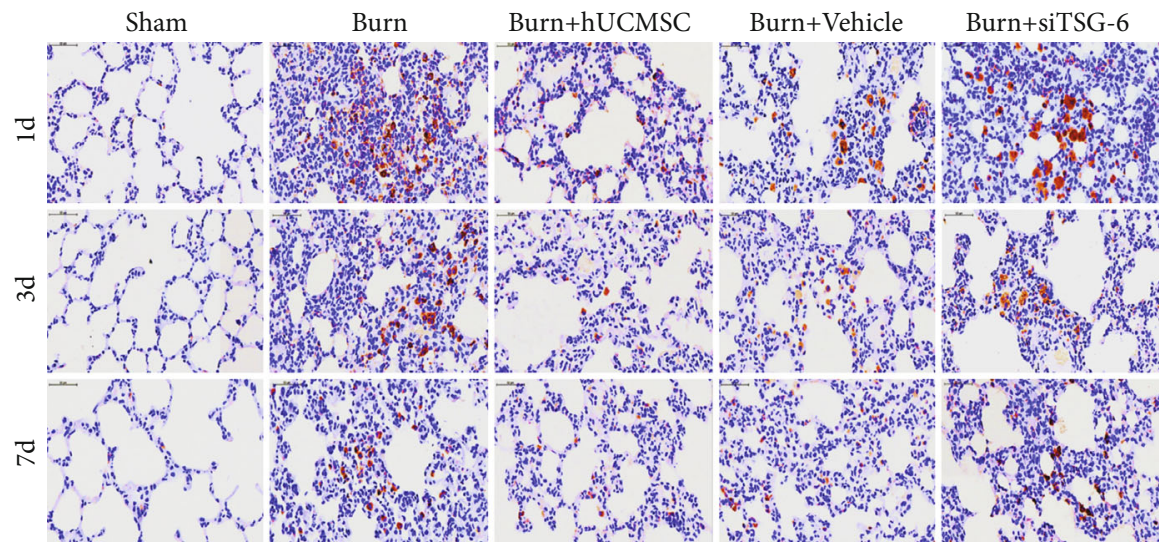

(a)

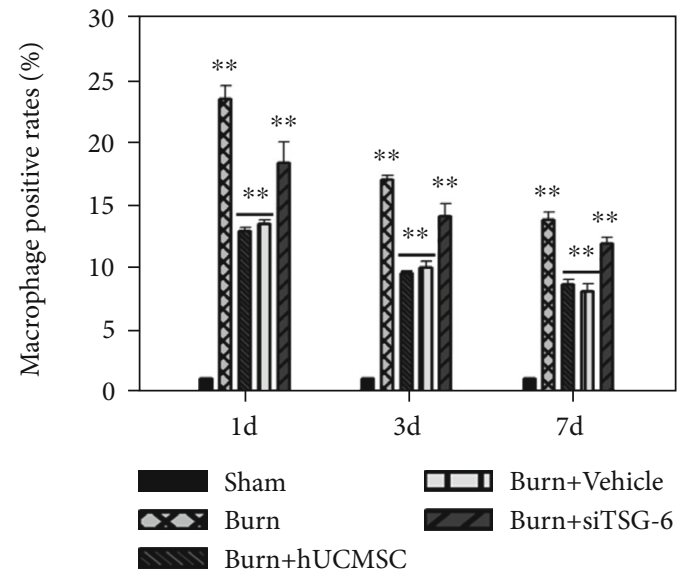

(b)

FIGURE 4: Effects of TSG-6 secretion and knockdown on macrophage infiltrations of lung tissues induced by severe burn. (a) Representative photographs of $\mathrm{CD}^{+} 8^{+}$in lung tissues using immumohistochemical staining are shown (the light microscope, 400x). (b) The quantitative analysis is shown in the corresponding histogram. Values are represented as mean $\pm \mathrm{SD}(n=6)$. Asterisk $(*)$ and double asterisk $(* *)$ stand for $p<0.05$ and $p<0.01$, respectively.

agreement with previous studies demonstrating that BMMSCs can reduce TNF- $\alpha$ and IL-6 secretion by lung macrophages via the paracrine pathway or direct contact with host cells [30]. Our results also confirmed that hUC-MSCs were able to protect the lung from injury through reducing the inflammatory response.

Interleukin-10 (IL-10), one of the most important antiinflammatory cytokines, is known to reduce the synthesis of proinflammatory cytokines. IL-10 treatment is supposed to be a promising therapeutic strategy to reduce lung injury [30-32]. Some argued that the progression of ALI is associated with decreased expression and secretion of IL-10 [33]. However, in our study, the IL-10 level rose in lung tissues after burn injury, and it was further markedly elevated after hUC-MSC administration. Thus, the protective effect of IL10 in lung inflammation response, which had been well described in previous studies, might partially explain the mechanism through which hUC-MSCs exerted their therapeutic effects on severe burn-induced ALI.

According to the above findings, it can be inferred that hUC-MSC administration improved ALI by balancing the homeostasis of the cytokine network. Furthermore, we would like to know more about how hUC-MSCs did work. In our previous study, we found that the TSG-6 levels were significantly elevated in the systemic inflammatory response in burn rats. TSG-6 expression is induced as a result of an inflammatory response. Our data showed that severe burninduced ALI could upregulate the expression of TSG-6, which was in agreement with our previous study [11]. The even higher level of TSG-6 in the burn+hUC-MSC group might relate to the TSG-6 secreted by hUC-MSCs in response to inflammatory signals. To test our hypothesis and investigate the role of TSG-6, TSG-6 knockdown was achieved via RNA interference by transfection with siRNA for TSG-6 in our ALI rat model. In the burn+siTSG-6 group, the level of TSG-6 was significantly decreased. Meanwhile, pathological changes and inflammatory response were detected in the burn+siTSG-6 group and burn+vehicle group. These results showed that the efficacies of hUCMSC implantation, including improvement of lung function and pulmonary metabolism function, structure protection of lung tissues, reduction of inflammatory cell infiltration, suppression of proinflammatory cytokines, and promotion of expression of anti-inflammatory, were diminished by TSG- 


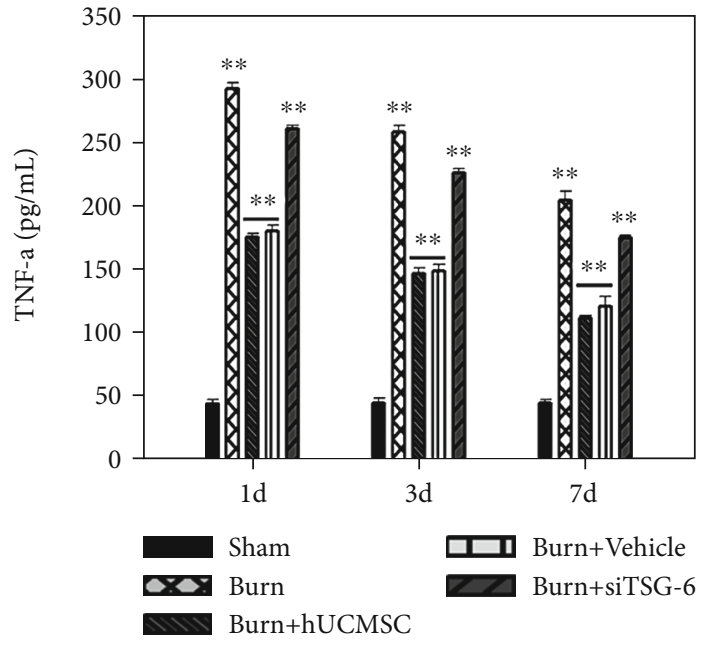

(a)

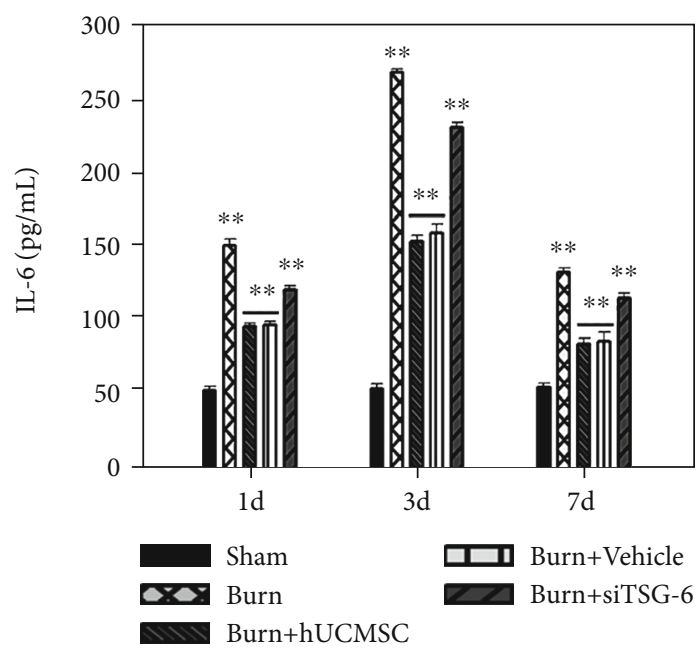

(c)

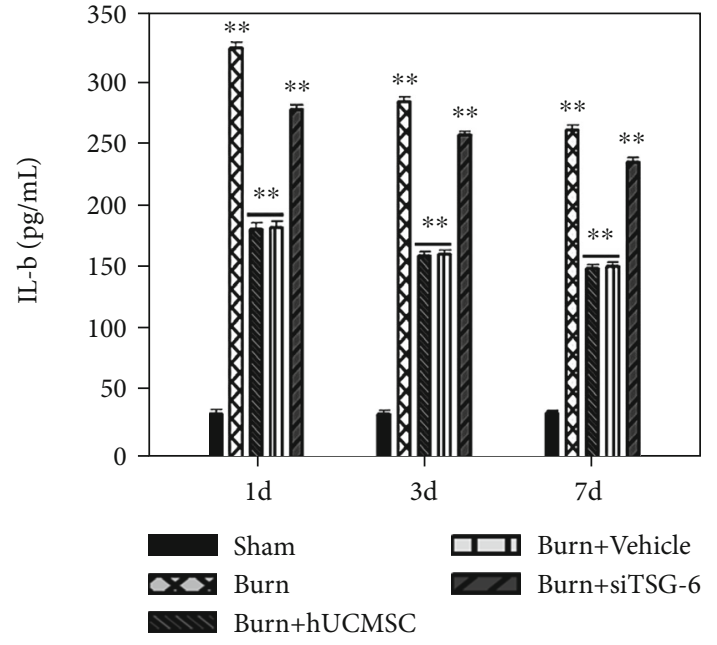

(b)

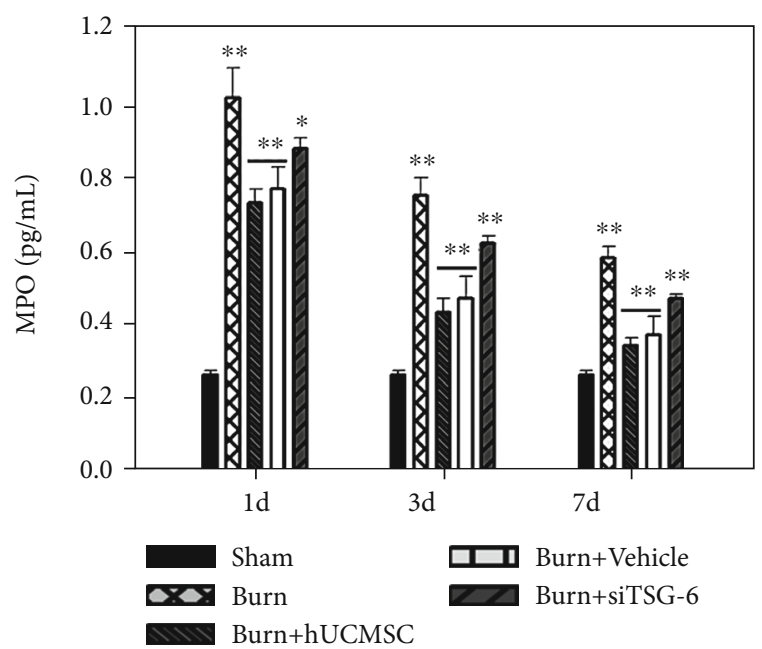

(d)

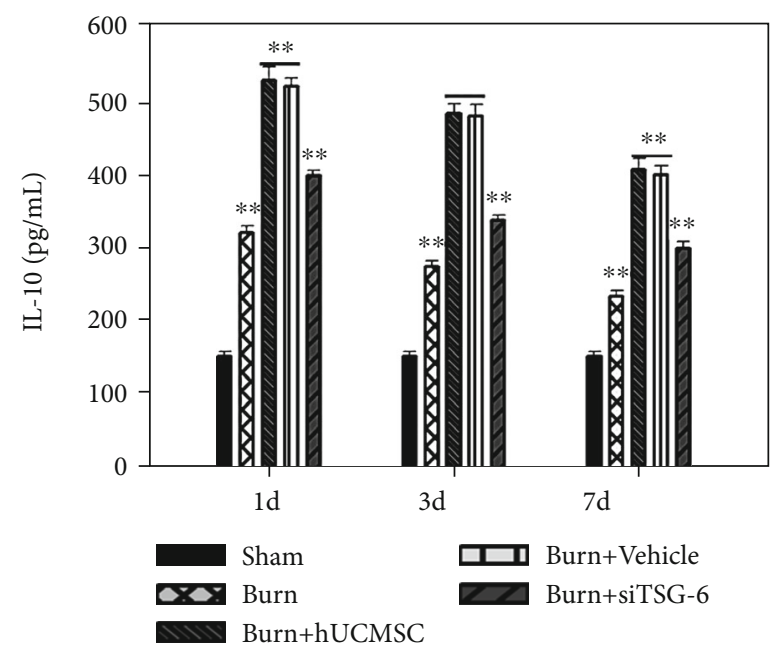

(e)

FIGURE 5: Effect of TSG-6 secretion and knockdown on inflammatory cytokines of lung tissues induced by severe burn. The effects of TSG-6 secretion and knockdown on TNF- $\alpha$ (a), IL-1 $\beta$ (b), IL-6 (c), MPO (d), and IL-10 (e) levels of lung tissue at $1 \mathrm{~d}, 3 \mathrm{~d}$, and $7 \mathrm{~d}$ postsevere burn, respectively. Values are represented as mean $\pm \mathrm{SD}(n=6)$. Asterisk $(*)$ and double asterisk $(* *)$ stand for $p<0.05$ and $p<0.01$, respectively. 
6 knockdown. Our data inferred that the anti-inflammatory properties of hUC-MSCs in severe burn-induced ALI are explained, at least in part, by the activation of hUC-MSCs to secrete TSG-6. These researches further proved that TSG-6 is an inflammation-associated secreted protein that has been implicated as having important and diverse tissue protective and anti-inflammatory properties [34]. Our results showed the observed beneficial effects of hUCMSCs in animal models of ALI and suggest that the antiinflammatory properties of hUC-MSCs in the lung are explained, at least in part, by the activation of hUC-MSCs to secrete TSG-6. TNF $\alpha$-stimulated gene-6 (TSG-6), a $30 \mathrm{kDa}$ protein generated by activated macrophages, modulates inflammation; however, its mechanism of action and role in the activation of macrophages were not fully understood. From our earlier report [11] and some literatures [35], we could find that TSG-6 inhibited the association of TLR4 with MyD88, thereby suppressing NF- $\kappa \mathrm{B}$ activation. TSG6 also prevented the expression of proinflammatory proteins (iNOS, IL-6, TNF- $\alpha$, IL- $1 \beta$, and CXCL1) while increasing the expression of anti-inflammatory proteins (CD206, Chi313, IL-4, and IL-10) in macrophages. This shift was associated with suppressed activation of proinflammatory transcription factors STAT1 and STAT3. Thus, TSG-6 functions by converting macrophages from a proinflammatory to an antiinflammatory phenotype secondary to suppression of TLR4/ NF- $\kappa$ B signaling and STAT1 and STAT3 activation.

It was well known that delivering locally has the advantage of reaching the target organ directly with relatively small dosage, faster and stronger effectiveness, and slighter systemic adverse reactions. In a clinical setting, physicians prefer to use medicine through intratracheal administration or aerosol inhalation for patients with lung diseases, especially for severe cases. Thus, we chose the intratracheal implantation as the route of medication. While increasing the dose of drugs might increase the efficacy of the treatment, we could not exclude systemic adverse reactions and more waste of resources caused by larger doses. Taking these factors into consideration, we selected the optimal dosage of hUC-MSCs $\left(1 \times 10^{6}\right)$ as suggested in our previous study [36].

In our rat model, full-thickness burn injury was established via placing the backside and abdomen into hot water, but not the chest, so ALI is caused by severe burn-induced excessive inflammation and serious metabolic disturbances rather than full-thickness escharotic burn at the back and abdomen.

Although the 50\% TBSA burn was severe, we managed to avoid the death of the rat model. In the early period of this study, we performed consistent treatments as follows: subjects were kept warm and then intraperitoneal injections of balanced salt solution $(40 \mathrm{ml} / \mathrm{kg})$ were immediately administered to prevent shock. And the burn wound was then treated with a $1 \%$ tincture of iodine and kept dry to prevent infection.

To the best of our knowledge, this is the first report about the therapeutic evaluation of hUC-MSCs in severe burn-induced ALI. TSG-6 secreted from hUC-MSCs played a key role in severe burn-induced ALI via inhibiting the inflammatory reaction in lung tissue. It suggests that intratracheal implantation of hUC-MSCs is an effective treatment for a severe burn-induced ALI rat model. Meanwhile, knockdown of TSG-6 mRNA expression in hUC-MSCs did not completely abrogate the anti-inflammatory effects suggesting that some other unknown mechanisms were involved. It means that additional experiments are required to determine the relative contribution of these factors to the beneficial effects of hUC-MSCs in lung injury.

\section{Abbreviation \\ TNF- $\alpha$ : Tumor necrosis factor $\alpha$ \\ TSG-6: $\quad$ TNF- $\alpha$ stimulated gene/protein 6 \\ hUC-MSC: Human umbilical cord-derived mesenchymal stem cell \\ ALI: $\quad$ Acute lung injury \\ ELISA: Enzyme-linked immunosorbent assay \\ ARDS: $\quad$ Acute respiratory distress syndrome \\ MSCM-sf: Mesenchymal stem cell medium serum-free medium \\ GFP: $\quad$ Green fluorescent protein \\ MPO: $\quad$ Myeloperoxidase \\ CD68: $\quad$ Cluster of differentiation 68 \\ siRNA: $\quad$ Small interference RNA \\ PMSF: $\quad$ Phenylmethanesulfonyl fluoride \\ IL: Interleukin.}

\section{Data Availability}

All authors are sure that all data and materials as well as software application or custom code support our published claims and comply with field standards. All materials in the manuscript including figures, tables, or text passages are originated from the authors.

\section{Ethical Approval}

The present study was approved by the Fourth Medical Center of PLA General Hospital Ethics Committees. All experimental procedures were in accordance with the international guidelines for biomedical research in experimental animals issued by CIOMS. After sample collection, rats were euthanized following the AVMA Guidelines for the Euthanasia of Animals: 2013 Edition.

\section{Disclosure}

We acknowledged that an identical manuscript was presented as a preprint version according to the following link: https://www.researchsquare.com/article/rs-127492/v1, due to the absence of peer reviews and official publication.

\section{Conflicts of Interest}

The authors declare that they have no competing interests. 


\section{Authors' Contributions}

All authors contributed to the study conception and design. Material preparation, data collection, and analysis were performed by Lingying Liu, Yu Wang, Yanan Liu, and Zhongyuan Li. The first draft of the manuscript was written by Xiaohong $\mathrm{Hu}$ and $\mathrm{Yu}$ Wang. The manuscript was revised by Xiaohong $\mathrm{Hu}$ and Yonghui Yu. All authors read and approved the final manuscript.

\section{Acknowledgments}

We thank Dr. Jing Yang, Xiao Li, Longlong Yang, and Shunyi Nie for animal experimental support. We thank Mr. Leo Liu for language modification. We acknowledge the grant support from the National Natural Science Foundation of China (NSFC 81701900 and 81571894).

\section{References}

[1] G. Bellani, J. G. Laffey, T. Pham et al., "Epidemiology, patterns of care, and mortality for patients with acute respiratory distress syndrome in intensive care units in 50 countries," JAMA, vol. 315, no. 8, pp. 788-800, 2016.

[2] S. Han and R. K. Mallampalli, "The acute respiratory distress syndrome: from mechanism to translation," Journal of Immunology, vol. 194, pp. 855-860, 2015.

[3] J. Bordes, G. Lacroix, P. Esnault et al., "Comparison of the Berlin definition with the American European consensus definition for acute respiratory distress syndrome in burn patients," Burns, vol. 40, no. 4, pp. 562-567, 2014.

[4] J. A. Shults, B. J. Curtis, M. M. Chen, E. B. O'Halloran, L. Ramirez, and E. J. Kovacs, "Impaired respiratory function and heightened pulmonary inflammation in episodic binge ethanol intoxication and burn injury," Alcohol, vol. 49, pp. 713-720, 2015.

[5] B. T. Thompson, R. C. Chambers, and K. D. Liu, "Acute respiratory distress syndrome," The New England Journal of Medicine, vol. 377, pp. 562-572, 2017.

[6] J. G. Laffey and M. A. Matthay, "Fifty years of research in ARDS. Cell-based therapy for acute respiratory distress syndrome. Biology and potential therapeutic value," American Journal of Respiratory and Critical Care Medicine, vol. 196, pp. 266-273, 2017.

[7] H. Hackstein, A. Lippitsch, P. Krug et al., "Prospectively defined murine mesenchymal stem cells inhibit Klebsiella pneumoniae-induced acute lung injury and improve pneumonia survival," Respiratory Research, vol. 16, no. 1, p. 123, 2015.

[8] L. Pedrazza, A. A. Cunha, C. Luft et al., "Mesenchymal stem cells improves survival in LPS-induced acute lung injury acting through inhibition of NETs formation," Journal of Cellular Physiology, vol. 232, no. 12, pp. 3552-3564, 2017.

[9] R. El Omar, J. Beroud, J. F. Stoltz, P. Menu, E. Velot, and V. Decot, "Umbilical cord mesenchymal stem cells: the new gold standard for mesenchymal stem cell-based therapies?," Tissue Engineering. Part B, Reviews, vol. 20, pp. 523-544, 2014.

[10] M. Colicchia, D. A. Jones, A. M. Beirne et al., "Umbilical cordderived mesenchymal stromal cells in cardiovascular disease: review of preclinical and clinical data," Cytotherapy, vol. 21, no. 10, pp. 1007-1018, 2019.
[11] L. Liu, H. Song, H. Duan et al., "TSG-6 secreted by human umbilical cord-MSCs attenuates severe burn-induced excessive inflammation via inhibiting activations of P38 and JNK signaling," Scientific Reports, vol. 6, no. 1, article 30121, 2016.

[12] L. Y. Liu, Y. S. Hou, J. K. Chai et al., "Basic fibroblast growth factor/vascular endothelial growth factor in the serum from severe burn patients stimulates the proliferation of cultured human umbilical cord mesenchymal stem cells via activation of Notch signaling pathways," Journal of Trauma and Acute Care Surgery, vol. 75, no. 5, pp. 789-797, 2013.

[13] H. L. Walker and A. D. Mason Jr., "A standard animal BURN," The Journal of Trauma, vol. 8, no. 6, pp. 1049-1051, 1968.

[14] X. Bai, L. Fan, T. He et al., "SIRT1 protects rat lung tissue against severe burn-induced remote ALI by attenuating the apoptosis of PMVECs via p38 MAPK signaling," Scientific Reports, vol. 5, no. 1, article 10277, 2015.

[15] E. M. Clary, E. K. O'Halloran, S. G. de la Fuente, and S. Eubanks, "Videoendoscopic endotracheal intubation of the rat," Laboratory Animals, vol. 38, pp. 158-161, 2004.

[16] K. Konno, Y. Shiotani, N. Itano, T. Ogawa, M. Hatakeyama, and K. Shioya, "Visible, safe and certain endotracheal intubation using endoscope system and inhalation anesthesia for rats," The Journal of Veterinary Medical Science, vol. 76, no. 10, pp. 1375-1381, 2014.

[17] Y. Yu, L. Yang, S. Han et al., "MIR-190B alleviates cell autophagy and burn-induced skeletal muscle wasting via modulating PHLPP1/Akt/FoxO3A signaling pathway," Shock, vol. 52, no. 5, pp. 513-521, 2019.

[18] S. Shin, Y. Kim, S. Jeong et al., "The therapeutic effect of human adult stem cells derived from adipose tissue in endotoxemic rat model," International Journal of Medical Sciences, vol. 10, no. 1, pp. 8-18, 2013.

[19] H. Zhu, Y. Xiong, Y. Xia et al., "Therapeutic Effects of Human Umbilical Cord-Derived Mesenchymal Stem Cells in Acute Lung Injury Mice," Scientific Reports, vol. 7, no. 1, p. 39889, 2017.

[20] H. Loy, D. I. T. Kuok, K. P. Y. Hui et al., “Therapeutic implications of human umbilical cord mesenchymal stromal cells in attenuating influenza a(H5N1) virus-associated acute lung injury," The Journal of Infectious Diseases, vol. 219, no. 2, pp. 186-196, 2019.

[21] S. C. Abreu, D. J. Weiss, and P. R. Rocco, "Extracellular vesicles derived from mesenchymal stromal cells: a therapeutic option in respiratory diseases?," Stem Cell Research \& Therapy, vol. 7, no. 1, p. 53, 2016.

[22] M. Gnecchi, P. Danieli, G. Malpasso, and M. C. Ciuffreda, "Paracrine mechanisms of mesenchymal stem cells in tissue repair," Methods in Molecular Biology, vol. 1416, pp. 123146, 2016.

[23] F. F. Cruz and P. R. M. Rocco, "Stem-cell extracellular vesicles and lung repair," Stem Cell Investigation, vol. 4, p. 78, 2017.

[24] F. J. Vizoso, N. Eiro, S. Cid, J. Schneider, and R. Perez-Fernandez, "Mesenchymal stem cell secretome: toward cell-free therapeutic strategies in regenerative medicine," International Journal of Molecular Sciences, vol. 18, 2017.

[25] L. Liu, X. Li, J. Yang et al., "Comparison of systemic inflammation response and vital organ damage induced by severe burns in different area," International Journal of Clinical and Experimental Pathology, vol. 8, no. 6, pp. 6367-6376, 2015.

[26] J. Li, D. Li, X. Liu, S. Tang, and F. Wei, "Human umbilical cord mesenchymal stem cells reduce systemic inflammation and 
attenuate LPS-induced acute lung injury in rats," Journal of Inflammation, vol. 9, no. 1, p. 33, 2012.

[27] H. M. Liu, Y. T. Liu, J. Zhang, and L. J. Ma, "Bone marrow mesenchymal stem cells ameliorate lung injury through antiinflammatory and antibacterial effect in COPD mice," Journal of Huazhong University of Science and Technology. Medical Sciences, vol. 37, pp. 496-504, 2017.

[28] Y. Hao, Y. Ran, B. Lu et al., "Therapeutic effects of human umbilical cord-derived mesenchymal stem cells on canine radiation-induced lung injury," International Journal of Radiation Oncology • Biology • Physics, vol. 102, no. 2, pp. 407-416, 2018.

[29] X. Wang, J. Yan, X. Xu et al., "Puerarin prevents LPS-induced acute lung injury via inhibiting inflammatory response," Microbial Pathogenesis, vol. 118, pp. 170-176, 2018.

[30] Y. Y. Wang, X. Z. Li, and L. B. Wang, "Therapeutic implications of mesenchymal stem cells in acute lung injury/acute respiratory distress syndrome," Stem Cell Research \& Therapy, vol. 4, p. 45, 2013.

[31] H. D. Li, Q. X. Zhang, Z. Mao, X. J. Xu, N. Y. Li, and H. Zhang, "Exogenous interleukin-10 attenuates hyperoxia-induced acute lung injury in mice," Experimental Physiology, vol. 100, pp. 331-340, 2015.

[32] L. Nie, W. Wu, Z. Lu, G. Zhu, and J. Liu, "CXCR3 may help regulate the inflammatory response in acute lung injury via a pathway modulated by IL-10 secreted by CD $8+$ CD122+ regulatory T cells," Inflammation, vol. 39, pp. 526-533, 2016.

[33] J. Chen, Y. Shao, G. Xu et al., "Bone marrow-derived mesenchymal stem cells attenuate phosgene-induced acute lung injury in rats," Inhalation Toxicology, vol. 27, no. 5, pp. 254261, 2015.

[34] A. J. Day and C. M. Milner, "TSG-6: a multifunctional protein with anti-inflammatory and tissue-protective properties," Matrix Biology, vol. 78-79, pp. 60-83, 2019.

[35] M. Mittal, C. Tiruppathi, S. Nepal et al., "TNF $\alpha$-stimulated gene-6 (TSG6) activates macrophage phenotype transition to prevent inflammatory lung injury," Proceedings of the National Academy of Sciences of the United States of America, vol. 113, no. 50, pp. E8151-E8158, 2016.

[36] L. Liu, Y. Yu, Y. Hou et al., "Human umbilical cord mesenchymal stem cells transplantation promotes cutaneous wound healing of severe burned rats," PLoS One, vol. 9, no. 2, article 88348, 2014. 